\title{
IoT-Enabled Solid Waste Management in Smart Cities
}

\author{
S. Vishnu ${ }^{1}$ (D) S. R. Jino Ramson ${ }^{1,2,3, * \mathbb{D}}$, Samson Senith ${ }^{4}$, Theodoros Anagnostopoulos ${ }^{5}$ (D), \\ Adnan M. Abu-Mahfouz ${ }^{6}(\mathbb{D})$, Xiaozhe Fan ${ }^{2}$, S. Srinivasan ${ }^{3}\left(\mathbb{D}\right.$ and A. Alfred Kirubaraj ${ }^{4}$ (D) \\ 1 Vignan's Foundation for Science, Technology and Research, Guntur 522213, India; \\ vishnuvazhamkuzhiyil@gmail.com \\ 2 School of Engineering Technology, Purdue University, West Lafayette, IN 47907, USA; fan115@purdue.edu \\ 3 Saveetha School of Engineering, Saveetha University, Tamil Nadu 602105, India; \\ Srinivasans.sse@saveetha.com \\ 4 Karunya Institute of Technology and Sciences, Coimbatore 641114, India; senith@karunya.edu (S.S.); \\ alfred.kirubaraj@gmail.com (A.A.K.) \\ 5 Department of Business Administration, University of West Attica, 12243 Athens, Greece; \\ Theodoros.Anagnostopoulos@uniwa.gr \\ 6 Council for Scientific and Industrial Research (CSIR), Department of Electrical and Electronic \\ Engineering Science, University of Johannesburg, Johannesburg 0001, South Africa; a.abumahfouz@ieee.org \\ * Correspondence: jinoramson@gmail.com
}

check for updates

Citation: Vishnu, S.; Ramson, S.R.J.; Senith, S.; Anagnostopoulos, T.; Abu-Mahfouz, A.M.; Fan, Z.; Srinivasan, S.; Kirubaraj, A.A. IoT-Enabled Solid Waste Management in Smart Cities. Smart Cities 2021, 4, 1004-1017.

https: / / doi.org/

10.3390/smartcities4030053

Academic Editor: Luis Francisco Díez

Received: 25 May 2021

Accepted: 5 July 2021

Published: 14 July 2021

Publisher's Note: MDPI stays neutral with regard to jurisdictional claims in published maps and institutional affiliations.

Copyright: (c) 2021 by the authors. Licensee MDPI, Basel, Switzerland. This article is an open access article distributed under the terms and conditions of the Creative Commons Attribution (CC BY) license (https:// creativecommons.org/licenses/by/ $4.0 /)$.

\begin{abstract}
The Internet of Things (IoT) paradigm plays a vital role for improving smart city applications by tracking and managing city processes in real-time. One of the most significant issues associated with smart city applications is solid waste management, which has a negative impact on our society's health and the environment. The traditional waste management process begins with waste created by city residents and disposed of in garbage bins at the source. Municipal department trucks collect garbage and move it to recycling centers on a fixed schedule. Municipalities and waste management companies fail to keep up with outdoor containers, making it impossible to determine when to clean them or when they are full. This work proposes an IoT-enabled solid waste management system for smart cities to overcome the limitations of the traditional waste management systems. The proposed architecture consists of two types of end sensor nodes: PBLMU (Public Bin Level Monitoring Unit) and HBLMU (Home Bin Level Monitoring Unit), which are used to track bins in public and residential areas, respectively. The PBLMUs and HBLMUs measure the unfilled level of the trash bin and its location data, process it, and transmit it to a central monitoring station for storage and analysis. An intelligent Graphical User Interface (GUI) enables the waste collection authority to view and evaluate the unfilled status of each trash bin. To validate the proposed system architecture, the following significant experiments were conducted: (a) Eight trash bins were equipped with PBLMUs and connected to a LoRaWAN network and another eight trash bins were equipped with HBLMUs and connected to a Wi-Fi network. The trash bins were filled with wastes at different levels and the corresponding unfilled levels of every trash bin were monitored through the intelligent GUI. (b) An experimental setup was arranged to measure the sleep current and active current contributions of a PBLMU to estimate its average current consumption. (c) The life expectancy of a PBLMU was estimated as approximately 70 days under hypothetical conditions.
\end{abstract}

Keywords: Internet of Things; solid waste management; trash bin; LoRaWAN; Wi-Fi; smart city; remote monitoring

\section{Introduction}

The Internet of Things (IoT) is a concept that refers to the ever-expanding network of internet-connected devices that are currently in use all over the world. Despite the current Covid-19 pandemic, the Internet of Things industry is growing, and it is estimated that around 30 billion IoT connections will exist by the end of 2025 [1]. Advanced smart sensors, cloud computing, big data, lightweight communication protocols, open-source 
server programs, and web development tools are the enabling technologies that accelerate the development and deployment of domain-specific IoT systems [2]. These interconnected devices can bridge the gap between the physical and digital worlds to enhance life, culture, and productivity. IoT has already exhibited promising approaches towards domain-specific applications such as Smart Homes [3], Smart City [4], Agriculture [5], Wearables [6,7], Smart Grids [8], Industrial Internet Telehealth [9], and Smart Supply chain Management [10].

IoT plays a pivotal role in enhancing smart city applications through real-time monitoring and management of city processes. One of the biggest challenges associated with smart city applications is solid waste disposal, which impacts our society's health and nature. Solid wastes are produced as a result of human and animal activities and are typically discarded as useless [11]. Annually, the world produces 2.01 billion tons of urban solid waste, with at least 33\% not being handled in an environmentally friendly way. By 2050, global waste is estimated to reach 3.40 billion tons, more than doubling population growth over that period [12].

The conventional waste management process begins with waste being generated by residents in cities and disposed of in trash bins at the point of creation. At a predetermined schedule, municipal department trucks gather the garbage and transport it to the recycling centers. Municipalities and corporations struggle to keep up with the outdoor bins to determine when to clean them or whether they are completely filled or not. One of the most pressing issues of our time is the prevention, tracking, and treatment of these wastes [13]. The conventional method of manually inspecting waste in bins is a time-consuming procedure that requires more human labor, time, and money which can be eliminated with today's technology [14].

Several WSNand IoT-based remote monitoring systems have been developed and deployed to address the aforementioned limitations of conventional waste management systems $[15,16]$. For tracking the bins, some of the monitoring systems used short-range wireless networking techniques including Bluetooth, Infrared, ZigBee, and Wi-Fi [17-19]. Similarly, a few works described smart bin monitoring systems that employs wide area network technologies such as NB-IoT, Sigfox, and LoRa [20-22].

As most homes are equipped with wireless internet connections, it is inferred that the Wi-Fi-based solution is well suited for monitoring the household bins. This will minimize the additional infrastructure expense. However, it is unsuitable to monitor trash bins in public places. Similarly, LoRa-based monitoring techniques are appropriate for monitoring bins in public areas. However, these methods are not preferable for monitoring bins at home because they would incur additional costs for the implementation of gateways and other facilities. According to the literature, none of the current solid waste management methods discuss the need for a hybrid architecture to efficiently manage solid waste in smart cities. Therefore, this work proposes an IoT-based solid waste management system for smart cities. The main contributions of this work in contrast to the existing solutions are as follows.

- Hybrid network architecture to monitor the household and public trash bins.

- $\quad$ Solar energy harvesting facility to extend the life time of the end nodes.

- A GPS module is embedded to evaluate the Geo-location of the trash bins

- An intelligent GUI is employed to view the status of every trash bin.

The remainder of this article is organized as follows. Section 2 presents the related work. Section 3 describes the proposed network architecture and Section 4 presents the results of the experiments performed. Finally, Section 5 concludes this article.

\section{Related Work}

Improper waste discharge, lack of systematic waste collection and management schemes, and ineffective waste management practices have all resulted in severe environmental issues and high waste disposal costs [23]. Because of the positive outcomes of IoT services, various waste management studies focused on IoT technologies have been undertaken by researchers to address the aforementioned issues involved in solid 
waste management. Construction industries, food processing industries, etc. consistently produce a portion of waste that has notable residue enhancing the significance of the application of waste management practices and sustainability principles [24].

Several works have been published that cover various aspects of waste management technology. A basic framework that recognizes the saturation of trash bins is presented in [25], in which the gathered data are transmitted via a wireless mesh network to conserve power and increase operating time. Besides, the smart bin employs a duty cycle strategy to cut down on power demand and increase operating time. For experiment validation, this approach was tested in an outdoor environment, which demonstrated the feasibility of the system. However, the system still has some unclear issues with the implementation. In [26], a food waste collection system in which data were gathered using radio-frequency identification (RFID) technology and distributed through a wireless mesh network is presented. However, the system fails to address the data gathering possibilities from the trash bins which are located in far places as cities cover a wide area. An automated line-following automobile with a robotic hand for waste collection is proposed in [27], but it lacks any algorithms to make the waste assemblage more efficient. An intelligent bin emphasized system is proposed in [28]. To prevent waste disposal outside the bin, this work recommended a method in which monitoring happens not only within the bin but also in the immediate area around it. Infrared sensors are mounted in the bins, which sense discarded garbage from a bin as well as to measure the bin's filled level.

A waste treatment and management system based on LoRaWAN technology is proposed in [29], a path optimization for the waste collection trucks is also mentioned in this work. Furthermore, an IoT-based network framework is proposed, but it did not offer lucidity about coordination and optimization for all garbage bins in the system. Another work in [30] developed a working prototype of smart trash bins. The proposed work focuses on segregating different forms of waste for better treatment and recycling. An integrated GPS module provides geotagging to the bins, a gas detector that senses hazardous gases, an infrared sensor that determines the filled level of the bin, a sound sensor for noise emission control, and a temperature and humidity sensor are all used with each bin. Sensors are linked to a microcontroller, which collect data and send it to a gateway through a LoRa transceiver module. The sensor data from multiple garbage bins will be received using a gateway module with a LoRa transceiver. The data will be processed locally, and the processed data will be sent to the cloud via TCP/IP and the MQTT protocol. The cloud server is enabled in such a way that alerts can be sent according to the waste levels of the bins. Furthermore, an AI-based algorithm runs to provide an optimized route for the waste-collecting trucks. An automated cloud-based sensing framework with mobile app-based control is presented in [31]. This work's unique feature is the ability to detect foul gases as well as the amount of waste present in the bins. The proposed work in [32] suggested an intelligent trash bin monitoring system that aims to detect the filled levels of the bins and send them to a central monitoring station at far locations. This system was designed to learn from previous experiences and forecast future states based on variables such as traffic congestion in the environment where the waste bins are located, cost efficiency balance, and other factors that are difficult for humans to observe and analyze.

From the literature, it is evident that the current systems are not covering all aspects for a full-fledged deployment of smart waste management systems in smart cities. The sensors and communication technologies adopted for the network architectures are to be evaluated in real-time for the feasibility of the developed systems. The frameworks need to be designed to manage household bins and public bins in smart cities. Moreover, the sensing nodes need to be power efficient and self-powered under long-term monitoring aspects of smart waste management systems.

\section{Network Architecture of the Developed System}

The network architecture of the developed IoT enabled solid waste management system is shown in Figure 1. It follows a hybrid network architecture to manage the trash 
bins in public places and residential areas effectively. The architecture comprises end sensor nodes, namely, Public Bin Level Monitoring Unit (PBLMU) and Home Bin Level Monitoring Unit (HBLMU) for monitoring the trash bins at public places and residential areas, respectively.

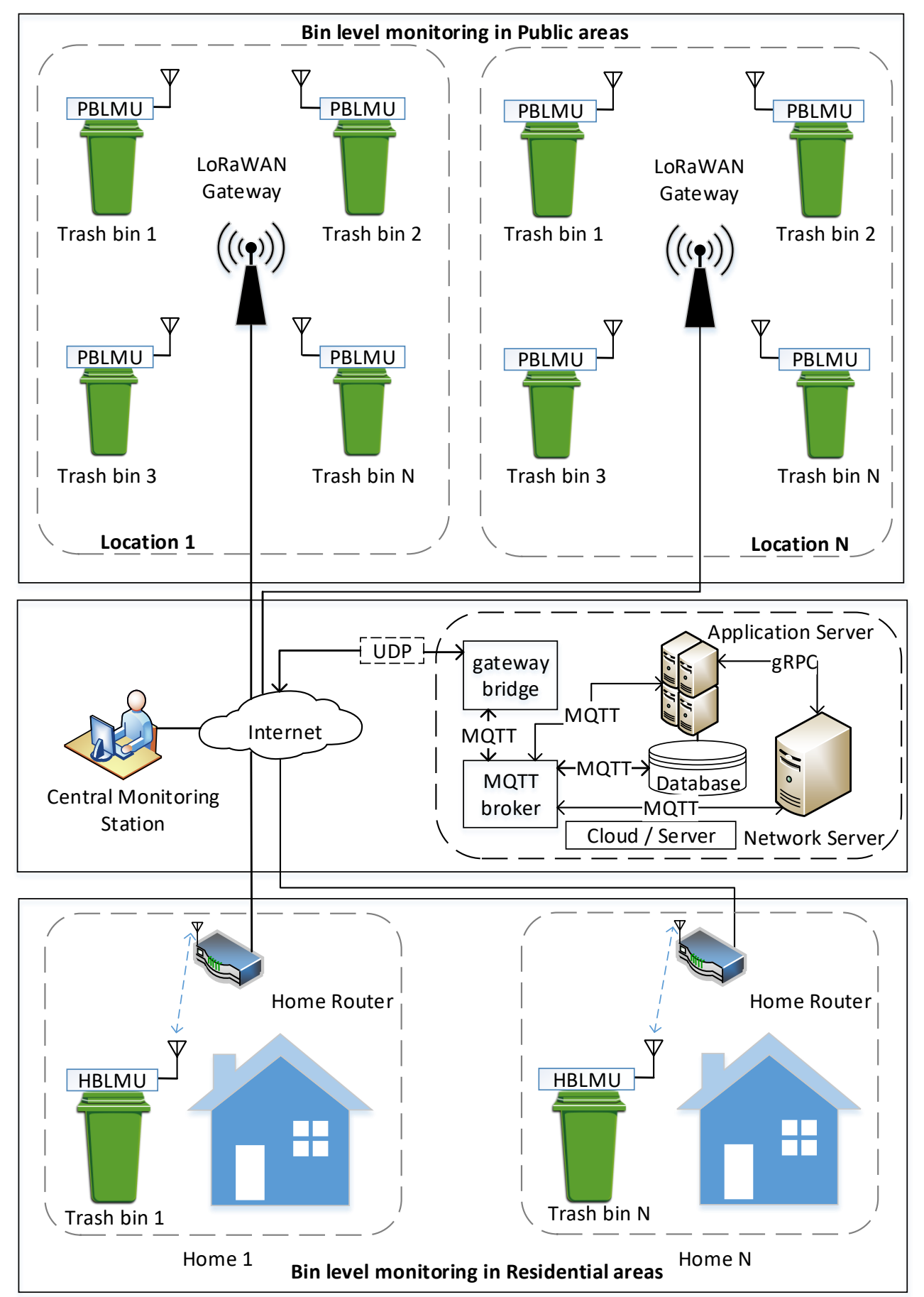

Figure 1. The network architecture of the developed system.

A LoRaWAN networking architecture is adopted for the deployment of PBLMUs, whereas a Wi-Fi-based communication is adopted for HBLMUs as the trash bins are associated with homes. The PBLMUs use a frequency of $915 \mathrm{MHz}$ to relay unfilled level and geo-location data from trash bins to the LoRaWAN gateway. The data from the PBLMUs are collected by the LoRaWAN gateway and uploaded to the server for storage and visualization, whereas a Wi-Fi module is integrated in the HBLMUs to get connected 
with the home routers for uploading data to the central monitoring station. The data are published into the server from the PBLMUs and HBLMUs through the MQTT broker which follows a publish-subscribe communication model. The PBLMUs and HBLMUs are the publishers that send data to the predefined topics of MQTT broker and the central monitoring server is the MQTT broker's subscriber, receiving data from it. The features such as low power consumption, rapid data transmission, lightweight nature, and ease of implementation make MQTT protocol very attractive for IoT-based remote monitoring systems. Through the intelligent GUI, the authorized persons can monitor and analyze the unfilled levels and the respective geo-locations of all the trash bins for the efficient waste collection.

\subsection{Design of the PBLMU}

The PBLMU is designed to collect the unfilled level and geo-locations of the trash bins located in public places. The block diagram of the designed PBLMU is shown in Figure 2. It comprises of an ultrasonic sensor and a GPS module to measure the unfilled level and the geo-location of the trash bins, respectively. A LoRa module is equipped with the PBLMU to establish the LoRaWAN network with the LoRaWAN gateway. Further, a power management unit is integrated to provide the required supply voltages to all the components of the PBLMU. Additionally, a solar panel is attached with the power management unit for energy harvesting and self-powering of PBLMUs since they are powered with batteries. A brief description of the components of the PBLMU is provided here.

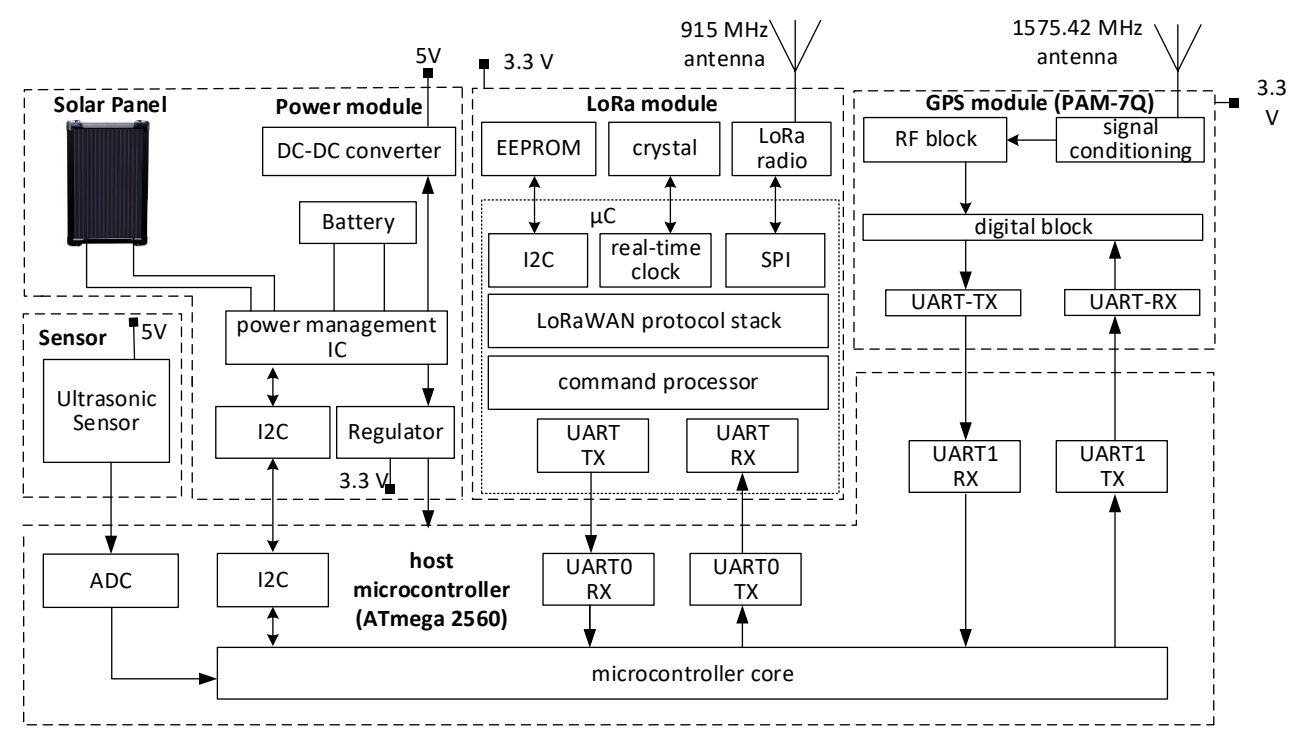

Figure 2. Block diagram of the Public Bin Level Monitoring Unit (PBLMU).

\subsubsection{Ultrasonic Sensor}

The ultrasonic sensor equipped in the PBLMU is a MB1010 LV-MaxSonar-EZ, which is lightweight, small in size, and commercially available. It is a cost-effective and dependable sensor with high accuracy, stable range detection, and a high-quality beam. With this sensor, high-frequency sound waves $(42 \mathrm{kHz})$ are produced, and the sensor evaluates the back-received echo. A wide range detection of solid waste ranging from $0 \mathrm{~m}$ to $6.45 \mathrm{~m}$ is possible with this sensor covering objects from very short to long range. This sensor is so accurate that there is no dead zone within the sensing range. The sensor outputs three different types of data at the same time: RS232 serial output, analog voltage output, and pulse width output. The sensor's actual operating temperature range is $-40{ }^{\circ} \mathrm{C}$ to $+65^{\circ} \mathrm{C}$ but the recommended temperature range is $0{ }^{\circ} \mathrm{C}$ to $+60{ }^{\circ} \mathrm{C}$. When the sensor is in triggered operation, it provides the desired reading range, allowing the unfilled level of a trash bin to be measured. A sensor operating in free-run mode, on the other hand, can continuously measure and output the range information. 


\subsubsection{GPS Module}

Several trash bins must be placed to manage solid waste over a large area. For the garbage truck to collect garbage, the geolocation coordinates for each trash bin is necessary. Manually recording the geolocation data of a large number of trash cans is a time-consuming task. Furthermore, the geolocation data aid in the identification of moved trash bins, stolen trash bins, as well as determining the shortest and most effective route for garbage collection. To collect the geolocation coordinates of each trash bin, the PBLMU is integrated with a PAM-7Q GPS antenna module. Embedded antenna, low power consumption, clear interface, high sensitivity of $-161 \mathrm{dBm}$, and sophisticated interference suppression are all features of the PAM-7Q, ensuring optimum efficiency even in hostile environments. The PAM-7Q's unique feature is its ability to achieve RHCP with a smaller patch antenna. Installation costs are kept to a minimum with the simple design and easy interfacing of an $18 \mathrm{~mm} \times 18 \mathrm{~mm}$ patch antenna. To minimize the PBLMU's average current consumption, the GPS module is configured in a power-saving mode called ON/OFF service.

\subsubsection{LoRa Module}

Each PBLMU is integrated with an RN2903 transceiver module to send data over a long distance while using minimal power. The RN2903 module achieves high interference immunity by using spread spectrum modulation. Every transmission is configured to use US902-928 MHz ISM band in a pseudo-random fashion to make the system more resistant to interference. The RN2903 module is operated by a $3.3 \mathrm{~V}$ DC supply and communicates with the host microcontroller using a UART. The RN2903 has a sensitivity of $-146 \mathrm{dBm}$ and a transmitting capacity of $18.5 \mathrm{dBm}$ that can be adjusted. It can also be programmed and managed over a UART port using ASCII commands. The RN2903 consumes $124 \mathrm{~mA}$ when transmitting at full power, and $13.5 \mathrm{~mA}$ in reception mode.

\subsubsection{Host Microcontroller}

The host microcontroller is the heart of the PBLMU; it is responsible for controlling all the functions of the PBLMU. In the PBLMU design, a high-performance, ultra-low-power, and advanced RISC architecture-based 8-bit Atmel ATmega 2560 microcontroller serve as the host microcontroller. The host microcontroller's peripheral features include four 8-bit PWM channels, a 16-channel ADC, four USARTs, a master/slave SPI serial port, and an I2C interface. The host microcontroller interfaces the MB1010 sensor via the ADC channel, the PAM-7Q GPS module via UART0, and the RN2903 LoRa transceiver module via UART1 in the PBLMU design. It runs at a clock frequency of $8 \mathrm{MHz}$ and has a $3.3 \mathrm{~V}$ operating voltage. Additionally, it has a temperature range comparable to that of the GPS module, which is $-40{ }^{\circ} \mathrm{C}$ to $85^{\circ} \mathrm{C}$.

\subsubsection{Power Management Unit}

The sensor requires a $5 \mathrm{~V}$ operating voltage, while the other components and the controller require a $3.3 \mathrm{~V}$ operating voltage. To meet the $5 \mathrm{~V}$ and $3.3 \mathrm{~V}$ requirements, a power management unit is integrated into the node design. The power management unit comprises of a solar panel, a battery of $2500 \mathrm{mAh}$ capacity, and a circuit for energy harvesting and battery charging. A low-power charger chip (BQ25505) is adopted to extract energy from the solar panel and to charge the batteries. It can obtain energy from a solar panel with a voltage as low as $100 \mathrm{mV}$. The BQ25505 features an ultralow quotient current consumption of $325 \mathrm{nA}$, integrated maximum power point monitoring from the solar panel and a battery health indicator. To prevent excess charging, the chip has a under-voltage threshold and over-voltage threshold of $3 \mathrm{~V}$ and $4.2 \mathrm{~V}$, respectively. The ultrasonic sensor is powered with a boost DC-DC converter (MCP16252T) as it requires a $5 \mathrm{~V}$ supply voltage. It operates in PFM/PWM mode automatically and achieves a typical efficiency of 96 percent. The MCP1825S LDO regulator keeps the voltage at $3.3 \mathrm{~V}$, to meet the power requirements of LoRa module, host microcontroller, and GPS module. 


\subsubsection{LoRaWAN Gateway}

Each PBLMU in the proposed IoT-enabled solid waste management system needs to be monitored from the central monitoring station. As the PBLMUs in a specific region create the Wide Area Network using non-IP-based communication protocols such as the LoRa protocol, each region requires a gateway to allow internet connectivity between the PBLMUs and the server. To function as the gateway in the proposed system, a commercially available microchip LoRa gateway is preferred. The LoRa gateway configures the network address, IP address, default subnet mask, server IP, server up port, and server down port in accordance with the system requirements. The LoRa radio board and the LoRa core board are the LoRa gateway's two primary components. The PBLMUs' data are received via the SMA radio connector on the radio board and fed to the RFSW1012-SPDT switch, which generates two independent RF outputs. Before demodulation, the RF outputs are filtered using two distinct frequencies. The microcontroller in the LoRa gateway's core board gathers data that have been transmitted by the radio board and then wraps the data in a JSON format prior to transmitting it to the Ethernet controller. Further, the Ethernet controller adds a UDP header to the packets, and these packets are sent to the server by way of a network switch.

\subsection{Design of the HBLMU}

Similarly, the HBLMU is designed to collect the unfilled level and geolocation of the trash bins installed at homes. The block diagram of the designed HBLMU is shown in Figure 3. It comprises an ultrasonic sensor and a GPS module to measure the unfilled level and the geolocation of the trash bins respectively. A Wi-Fi module is equipped with the HBLMU to establish wireless connectivity with the home router which is acting as the access point to get connected to the cloud server. Further, a power management unit is integrated to provide the required supply voltages to other components of the HBLMU. Additionally, a solar panel is attached with the power management unit for energy harvesting and self-powering of HBLMUs as they are powered with batteries. The functionality and specifications of the ultrasonic sensor, GPS module, and power management unit are alike to that in the design of PBLMU, the remaining components of the HBLMU are described here.

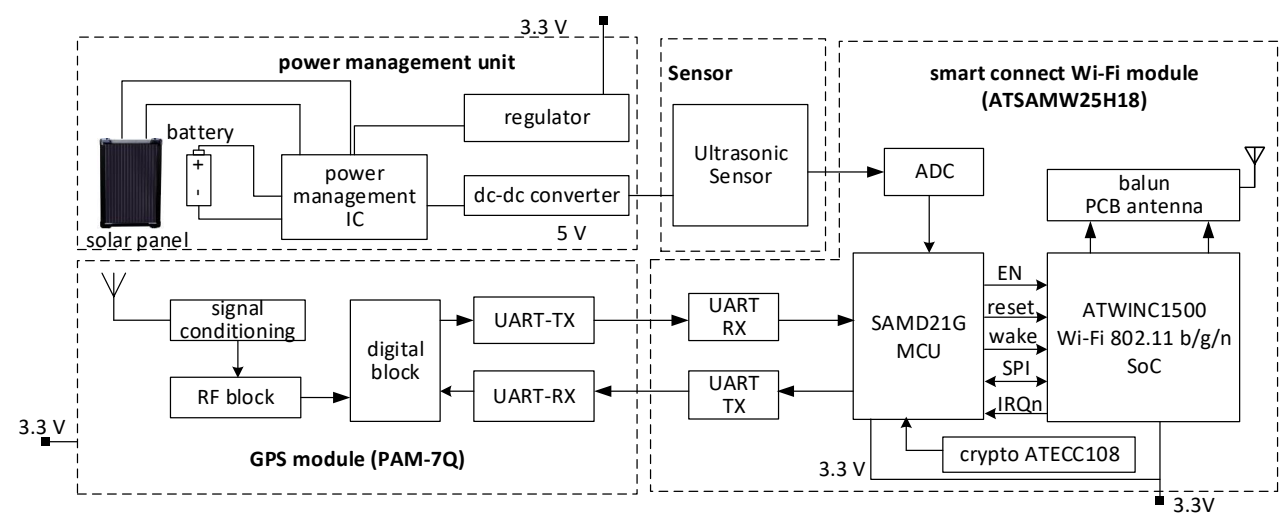

Figure 3. Block diagram of the HBLMU.

\subsubsection{Wi-Fi Module}

The Wi-Fi module in the HBLMU is a Microchip Smart Connect ATSAMW25H18 SOC. It is certified and incorporated with the $802.11 \mathrm{IP}$ stack. It is composed of an ARM cortex M0+ microcontroller (host computer) and an ATWINC1500 Wi-Fi network processor. The host machine operates at a $48 \mathrm{MHz}$ clock frequency. It includes an on-chip memory management engine that reduces the load on the host computer. It includes an $8 \mathrm{Mb}$ internal flash memory for firmware updates via OTA. Secure network access is established using TLS and SSL protocols. Additionally, it supports network protocols such as DHCP, DNS, 
UDP, HTTP, and HTTPS. The controller's operating voltage is normally $3.7 \mathrm{~V}$. It features a ten-bit DAC and a fourteen-channel 12-bit ADC. ATSAMW25H18 is the best option for the HBLMU design due to its extremely low power consumption, over-the-air software update capability, and built-in security features.

\subsubsection{Home Router}

Attributed to the reason that the HBLMUs are used to track the unfilled levels and geolocations of household trash bins, the home router is used to establish connectivity between the central monitoring station and sensing nodes. The wireless router used in this experiment is a TP-Link TL-MR6400. It complies with IEEE 802.11/n/a and IEEE $802.11 \mathrm{~b} / \mathrm{g} / \mathrm{n}$ wireless standards and supports data transfer rates of up to $300 \mathrm{Mbps}$.

\subsection{The Server and the GUI}

The server's hardware configuration includes an Intel Core i7-8700T processor, 16 GB RAM, and a 500 GB hard drive running Ubuntu 20.04 LTS. The software package provides open-source components such as Redis, PostgreSQL, ChirpStack gateway bridge, Eclipse Mosquitto, ChirpStack network server, and ChirpStack application server, as well as an intelligent GUI. The Eclipse Mosquitto message broker is used to implement the MQTT protocol, which uses a publish/subscribe model to transport data. Redis is an in-memory database used to store transient data, while PostgreSQL is used to store long-term data. The intelligent GUI is designed using the C Sharp programming language on the .NET platform. The .NET core 3.0 includes the gRPC framework, a lightweight and highly performant RPC framework that enables real-time message push without polling. After decoding and systematically storing the bin level data, the generated graphical representation of the main window, all area icons, and all trash bin icons are graphically mapped to the PBLMU and HBLMU measurements. To quickly identify the trash bin level, each level is assigned with a color code.

\section{Results and Discussion}

The fabricated HBLMU and PBLMU are shown in Figures 4 and 5, respectively. Significant experiments were conducted to validate the feasibility of the developed IoT-enabled solid waste management system. First, an experiment was conducted to validate the developed IoT system by monitoring the corresponding unfilled levels of the trash bins through the intelligent GUI. Second, an experimental setup was arranged to measure the sleep current and active current contributions of a PBLMU to obtain its average current consumption. Finally, the life expectancy of a PBLMU was estimated under hypothetical conditions.

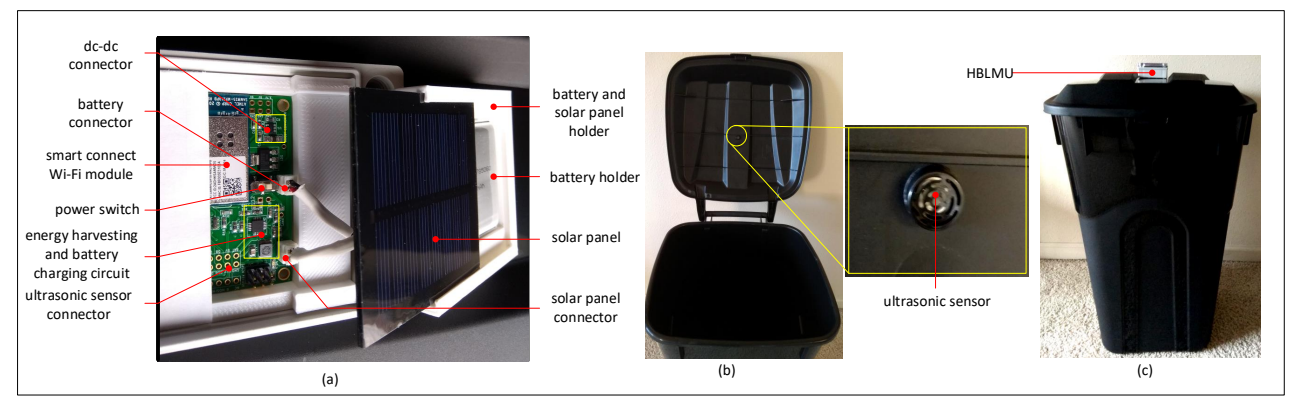

Figure 4. (a) Various components of the fabricated HBLMU. (b) Installation of HBLMU on a trash bin. (c) Front view of the HBLMU equipped trash bin. 


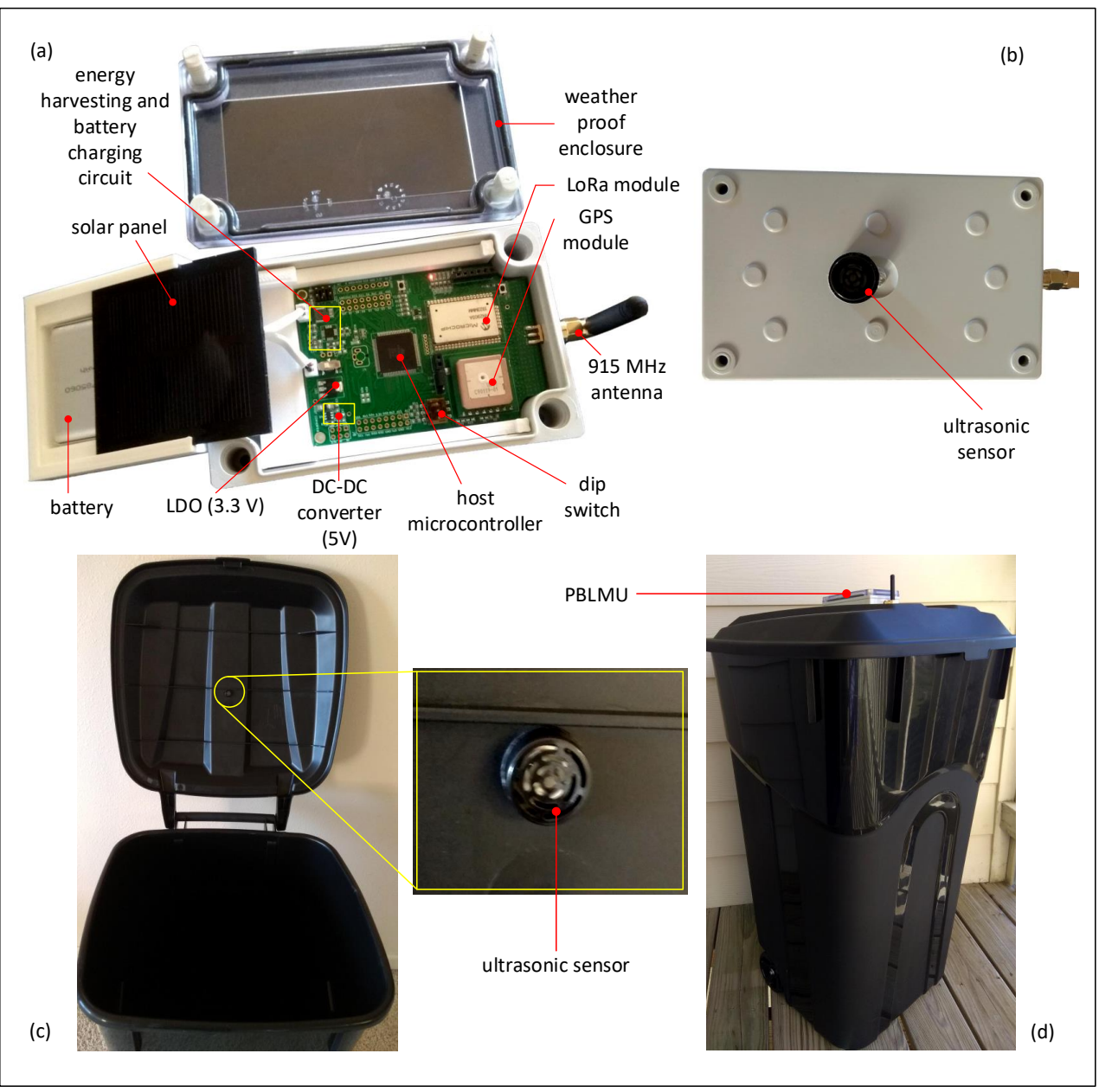

Figure 5. (a) Various components of the fabricated PBLMU. (b) Rear view of the fabricated PBLMU. (c) Installation of PBLMU on a trash bin. (d) Front view of the PBLMU equipped trash bin.

\subsection{Validation of the Developed System}

The trash bins used in our study have dimensions of $40 \mathrm{~cm} \times 40 \mathrm{~cm}$ on the top, $30 \mathrm{~cm} \times 30 \mathrm{~cm}$ on the base, and $82.5 \mathrm{~cm}$ in height. An experiment was carried out in both indoor and outdoor environments to validate the developed IoT system, where 8 bins were installed with PBLMUs and connected to a LoRaWAN, and another 8 bins were installed with HBLMUs and connected to a Wi-Fi network, respectively. The firmware of the PBLMU and the HBLMU were programmed to send unfilled data every $5 \mathrm{~min}$. The trash bins were filled with paper, card boxes, bottles, and clothes at different levels, and the corresponding unfilled levels of the trash bins were monitored in the Intelligent GUI.

Taking into account the readings from the PBLMU, HBLMU, and the trash bin's maximum unfilled level, the intelligent GUI assigns a color code to each trash bin. Table 1 shows the threshold unfilled levels of the trash bins for various color codes.

Table 1. Mapping table for the threshold unfilled levels and color code.

\begin{tabular}{ccc}
\hline Unfilled Bin Level in cm & Color Code & Status \\
\hline Equal to 82.5 & Green & Empty \\
Greater than 70 & Green & Lightly filled \\
Between 30 and 70 & Orange & Partially filled \\
Less than 30 & Red & Almost full \\
\hline
\end{tabular}

Figure 6 illustrates a screenshot of the developed intelligent GUI, which depicts the global level (home icon), levels of different regions, and Region 1's trash bin levels. 
The intelligent GUI was designed in a hierarchical fashion to allow for real-time monitoring of the trash bin's exact level and location. The intelligent GUI's primary icon (at the top level) is a progressive bar that is synchronized with all PBLMUs and HBLMUs in the IoT-enabled solid waste management system. The primary icon's color code corresponds to the trash bins lowest unfilled value. When the user clicks on the home icon, the intelligent GUI automatically displays the regional icons. Additionally, the region icon functions as a progressive bar whose status is determined by the number of unfilled PBLMUs and HBLMUs in that region. The color code assigned to the region icons corresponds to the lowest level of unfilled trash bins in that region. When a user clicks on a region icon, the intelligent GUI displays all trash bins in that region. Additionally, the trash bin icons function as a progressive bar whose status is determined by the sensor value of the PBLMU or HBLMU. From the illustration, the main icon is red because the unfilled value of trash bin 1 is $11.2 \mathrm{~cm}$, which is less than $30 \mathrm{~cm}$ (threshold value). This immediately alerts the user that some trash bins in the IoT-enabled solid waste management system are approaching capacity. The color of the region icons varies; Region 1 is red because the unfilled value of trash bin 1 is $11.2 \mathrm{~cm}$. Regions 2 and 3 are highlighted in orange because the trash bins' unfilled level is between 30 and $70 \mathrm{~cm}$. As the unfilled amount of all trash bins in Region 4 exceeds $70 \mathrm{~cm}$, the region is green in color. When a user hovers or clicks on a trash bin icon, the exact unfilled level of the bin as well as its geo-location coordinates are shown.

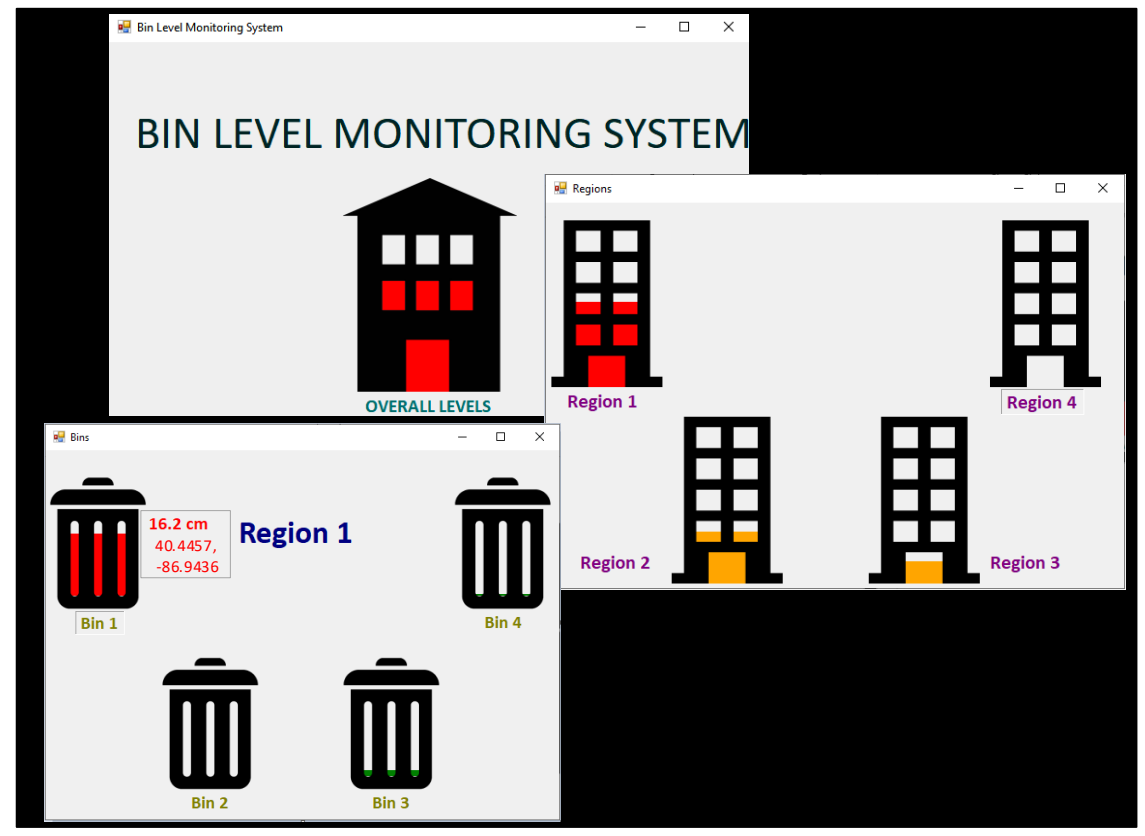

Figure 6. Screenshot of the Intelligent GUI.

\subsection{Average Current Consumption of a PBLMU}

We used an INA233 evaluation module to assess the active and sleep mode current contributions of a PBLMU while operating the LoRa module at a spreading factor of 7 , power level of $10 \mathrm{dBm}$, bandwidth of $125 \mathrm{KHz}$, and a coding rate of $4 / 5$. Table 2 shows the nomenclature of the mathematical symbols used in the equations. The measured current consumption in active and sleep modes are as follows.

PBLMU's active current contribution:

$$
\begin{aligned}
Q_{P B L M U_{a}} & =\left(\left[I_{E H C_{q}}+I_{L D O_{q}}+I_{D C-D C_{q}}+I_{H M_{a}}\right] \times T_{H M_{a}}\right)+ \\
& \left(I_{G P S_{a}} \times T_{G P S_{a}}\right)+\left(I_{L o R a_{a}} \times T_{L o R a_{a}}\right)+ \\
& \left(I_{\text {sensor }_{a}} \times T_{\text {sensor }_{a}}\right) \\
& =0.3316 \mathrm{~A} \times \mathrm{s}
\end{aligned}
$$


PBLMU's sleep current contribution:

$$
\begin{aligned}
Q_{P B L M U_{s}} & =\left(I_{E H C_{q}}+I_{L D O_{q}}+I_{D C-D C_{q}}+I_{H M_{s}}+I_{L o R a_{s}}\right) \times\left(T-T_{H M_{a}}\right) \\
& =0.1115 \mathrm{~A} \times s
\end{aligned}
$$

Average current consumption of a PBLMU:

$$
\begin{aligned}
I_{P B L M U} & =\frac{Q_{P B L M U_{a}}+Q_{P B L M U_{s}}}{T} \\
& =1.5 \mathrm{~mA}
\end{aligned}
$$

Table 2. Nomenclature of mathematical symbols.

\begin{tabular}{ll}
\hline Parameter & Description \\
\hline$I_{E H C_{q}}$ & Quiescent current of BQ25505 energy harvesting IC \\
$I_{L D O_{q}}$ & LDO's quiescent current \\
$I_{P B L M U}$ & PBLMU's average current consumption \\
$I_{D C-D C_{q}}$ & DC-DC converter's quiescent current \\
$Q_{B a t t e r y}$ & Capacity of battery \\
$I_{H M_{a}}$ & Host machine's current consumption in active mode \\
$P B L M U_{\text {days }}$ & PBLMU's life expectancy \\
$T_{H M_{a}}$ & Host machine's time period in active mode \\
$I_{G P S_{a}}$ & GPS module current consumption in active mode \\
$I_{L o R a_{a}}$ & LoRa's active current consumption \\
$I_{L o R a_{s}}$ & LoRa's sleep current consumption \\
$I_{S e n s o r a}$ & Sensor's active current consumption \\
$T_{G P S_{a}}$ & GPS module time period in active mode \\
$I_{H M_{s}}$ & Host machine's current consumption in sleep mode \\
$T_{L o R a_{a}}$ & LoRa's time period in active mode \\
$T_{S e n s o r a}$ & Sensor's time period in active mode \\
$T$ & Transmission time period \\
\hline
\end{tabular}

\subsection{Life Expectancy of a PBLMU}

The PBLMU's life expectancy was determined under the hypothetical scenario under which the battery's voltage is optimal before its power is depleted. Assume a standard battery has a capacity of $2500 \mathrm{mAh}$ and the following calculation is used to determine the life expectancy of the sensing nodes.

$$
\begin{aligned}
P_{B L M U_{\text {days }}} & =\frac{Q_{\text {Battery }}}{I_{P B L M U}} \\
& \approx 70 \text { days }
\end{aligned}
$$

Once the battery is fully charged it can power a PBLMU for approximately 70 days without any interruption.

\section{Conclusions}

The development and validation of a hybrid network architecture approach to efficiently manage trash bins in public places and residential areas of cities were discussed in this paper. All facets of an IoT system have been developed, including the design of end nodes, i.e., PBLMU and HBLMU; long-range data transmission with LoRa network for public places and Wi-Fi connectivity for homes; long-term data storage; and hierarchical visualization of trash bin level with the intelligent GUI. Experiments were conducted to validate the developed IoT system, as well as to estimate current consumption and maximum life expectancy of the end node. First, the trash bins had been filled with waste, and the corresponding unfilled levels on the Intelligent GUI were monitored. Second, based on the measured active and sleep current contributions, the PBLMU's average current 
consumption is calculated as $1.5 \mathrm{~mA}$. Finally, the life expectancy of a PBLMU was estimated as approximately 70 days under hypothetical conditions. According to the obtained results, the proposed IoT-enabled solid waste management system is well suited for monitoring real-time trash bin information in smart cities.

Future work in this area, trash bin information (unfilled level and geolocation coordinates) obtained through the proposed IoT system can be used for framing geographic information system (GIS). Furthermore, optimum routes can be obtained through machine learning algorithms for waste collection trucks.

Author Contributions: Investigation, S.V.; methodology, S.V., S.R.J.R.; Project administration, S.R.J.R.; Supervision, S.R.J.R.; Writing, review \& editing, S.V., S.R.J.R., S.S. (Samson Senith), T.A., A.M.A.-M., X.F., S.S. (S. Srinivasan) and A.A.K. All authors have read and agreed to the published version of the manuscript.

Funding: This research received no external funding.

Institutional Review Board Statement: Not applicable.

Informed Consent Statement: Not applicable.

Data Availability Statement: Not applicable.

Conflicts of Interest: The authors declare no conflicts of interest.

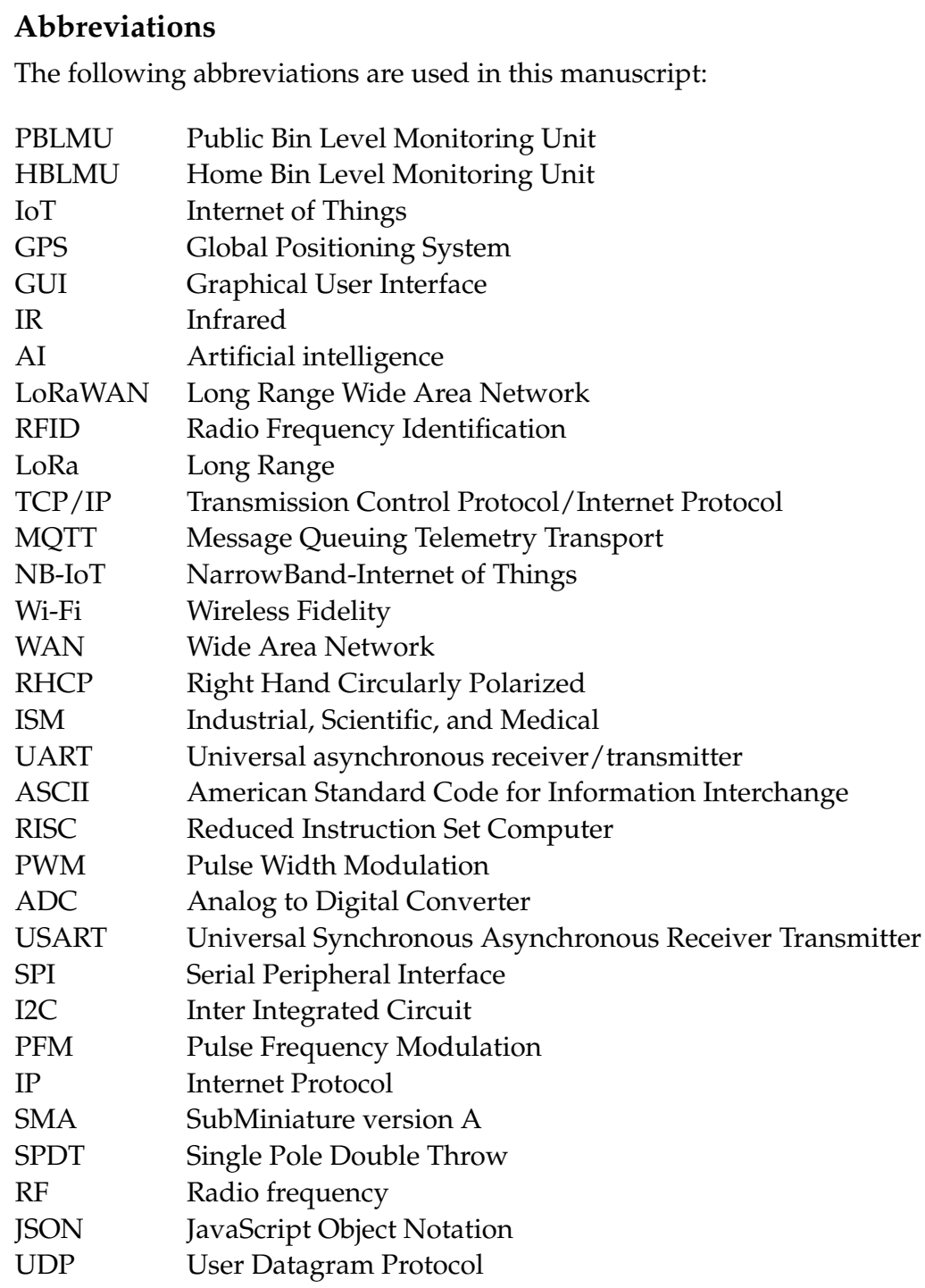




$\begin{array}{ll}\text { SOC } & \text { System on a Chip } \\ \text { OTA } & \text { Over-The-Air } \\ \text { TLS } & \text { Transport Layer Security } \\ \text { SSL } & \text { Secure Sockets Layers } \\ \text { DHCP } & \text { Dynamic Host Configuration Protocol } \\ \text { DNS } & \text { Domain Name System } \\ \text { HTTP } & \text { Hypertext Transfer Protocol } \\ \text { HTTPS } & \text { Hypertext Transfer Protocol Secure } \\ \text { DAC } & \text { Digital to Analog Converter } \\ \text { RPC } & \text { Remote Procedure Call } \\ \text { LDO } & \text { Low Dropout }\end{array}$

\section{References}

1. Song, Y.; Yu, F.R.; Zhou, L.; Yang, X.; He, Z. Applications of the Internet of Things (IoT) in Smart Logistics: A Comprehensive Survey. IEEE Internet Things J. 2020, 8, 4250-4274. [CrossRef]

2. Jino Ramson, S.R.; Vishnu, S.; Shanmugam, M. Applications of internet of things (IoT)—An Overview. In Proceedings of the 2020 5th International Conference on Devices, Circuits and Systems (ICDCS), Coimbatore, India, 5-6 March 2020.

3. Yang, Q.; Wang, H. Privacy-Preserving Transactive Energy Management for IoT-aided Smart Homes via Blockchain. arXiv 2021, arXiv:2101.03840.

4. Theodoros, A. IoT-enabled tip and swap waste management models for smart cities. Int. J. Environ. Waste Manag. 2020, accepted for publication.

5. Ramson, S.J.; León-Salas, W.D.; Brecheisen, Z.; Foster, E.J.; Johnston, C.T.; Schulze, D.G.; Málaga, M.P. A Self-Powered, Real-Time, LoRaWAN IoT-based Soil Health Monitoring System. IEEE Internet Things J. 2021, 8, 9278-9293. [CrossRef]

6. Vishnu, S.; Ramson, S.J.; Raju, K.L.; Anagnostopoulos, T. Simple-Link Sensor Network-Based Remote Monitoring of Multiple Patients. In Intelligent Data Analysis for Biomedical Applications; Academic Press: Cambridge, MA, USA, 2019 ; pp. $237-252$.

7. Vishnu, S.; Ramson, S.R.J.; Jegan, R. Internet of medical things (IoMT)—An overview. In Proceedings of the 20205 th International Conference on Devices, Circuits and Systems (ICDCS), Coimbatore, India, 5-6 March 2020.

8. Avancini, D.B.; Rodrigues, J.J.; Rabêlo, R.A.; Das, A.K.; Kozlov, S.; Solic, P. A new IoT-based smart energy meter for smart grids. Int. J. Energy Res. 2021, 45, 189-202. [CrossRef]

9. Vishnu, S.; Ramson, S.R.J. An Internet of Things Paradigm: Pandemic Management (incl. COVID-19). In Proceedings of the 2021 International Conference on Artificial Intelligence and Smart Systems (ICAIS), Coimbatore, India, 25-27 March 2021; pp. 1371-1375.

10. Abhishek, B. Blockchain \& IoT: A Paradigm Shift for Supply Chain Management. In Blockchain for Business: How It Works and Creates Value; Scrivener Publishing LLC: Austin, TX, USA, 2021; pp. 159-178.

11. Ramson, S.J.; Bhavanam, D.; Draksharam, S.; Kumar, R.; Moni, D.J.; Kirubaraj, A.A. Radio Frequency Identification and Sensor Networks based Bin Level Monitoring Systems-A Review. In Proceedings of the 2018 4th International Conference on Devices, Circuits and Systems (ICDCS), Coimbatore, India, 16-17 March 2018.

12. Kaza, S.; Yao, L.; Bhada-Tata, P.; Van Woerden, F. What a Waste 2.0: A Global Snapshot of Solid Waste Management to 2050; World Bank Publications: Washington, DC, USA, 2018.

13. Maria, C.; Góis, J.; Leitão, A. Challenges, perspectives, of, greenhouse, gases, emissions, from, municipal, solid, waste, management in Angola. Energy Rep. 2020, 6, 364-369. [CrossRef]

14. Venkiteela, L.K. Status and challenges of solid waste management in Tirupati city. Mater. Today Proc. 2020, 33, 470-474. [CrossRef]

15. Ramson, S.J.; Moni, D.J. Wireless sensor networks based smart bin. Comput. Electr. Eng. 2017, 64, 337-353. [CrossRef]

16. Prabakar, D.; Lakshmy, N.; Vishnu, S. Dynamic Channel State Information based Relay Selection in Device-to-Device Communication. In Proceedings of the 2020 International Conference on Electronics and Sustainable Communication Systems (ICESC), Coimbatore, India, 2-4 July 2020.

17. Ramson, S.J.; Moni, D.J. Applications of wireless sensor networks-A survey. In Proceedings of the 2017 International Conference on Innovations in Electrical, Electronics, Instrumentation and Media Technology (ICEEIMT), Coimbatore, India, 3-4 February 2017.

18. Suresh, N.; Limbo, A.; Hashiyana, V.; Ujakpa, M.M.; Nyirenda, C. An internet of things (IoT) based solid waste monitoring system. In Proceedings of the 2nd International Conference on Intelligent and Innovative Computing Applications, Online, 24-25 September 2020.

19. Ramson, S.J.; Moni, D.J.; Vishnu, S.; Anagnostopoulos, T.; Kirubaraj, A.A.; Fan, X. An IoT-based bin level monitoring system for solid waste management. J. Mater. Cycles Waste Manag. 2021, 23, 516-525. [CrossRef]

20. Harith, M.Z.M.Z.; Hossain, M.A.; Ahmedy, I.; Idris, M.Y.I.; Soon, T.K.; Noor, R.M. Prototype Development of IoT Based Smart Waste Management System for Smart City. IOP Conf. Ser. Mater. Sci. Eng. 2020, 884, 012051. [CrossRef]

21. Routray, S.K.; Sharmila, K.P.; Akanskha, E.; Ghosh, A.D.; Sharma, L.; Pappa, M. Narrowb and IoT (NBIoT) for Smart Cities. In Proceedings of the 2021 Third International Conference on Intelligent Communication Technologies and Virtual Mobile Networks (ICICV), Tirunelveli, India, 4-6 February 2021. 
22. Ramson, S.J.; Vishnu, S.; Kirubaraj, A.; Anagnostopoulos, T.; Abu-Mahfouz, A.M. A LoRaWAN IoT enabled Trash Bin Level Monitoring System. IEEE Trans. Ind. Inform. 2021. [CrossRef]

23. Gutberlet, Jutta, and Sayed Mohammad Nazim Uddin. Household waste and health risks affecting waste pickers and the environment in low-and middle-income countries. Int. J. Occup. Environ. Health 2017, 23, 299-310. [CrossRef] [PubMed]

24. Amaral, R.E.C.; Brito, J.; Buckman, M.; Drake, E.; Ilatova, E.; Rice, P.; Sabbagh, C.; Voronkin, S.; Abraham, Y.S. Waste Management and Operational Energy for Sustainable Buildings: A Review. Sustainability 2020, 12, 5337. [CrossRef]

25. Folianto, F.; Low, Y.S.; Yeow, W.L. Smartbin: Smart waste management system. In Proceedings of the 2015 IEEE Tenth International Conference on Intelligent Sensors, Sensor Networks and Information Processing (ISSNIP), Singapore, 7-9 April 2015.

26. Popa, C.L.; Carutasu, G.; Cotet, C.E.; Carutasu, N.L.; Dobrescu, T. Smart city platform development for an automated waste collection system. Sustainability 2017, 9, 2064. [CrossRef]

27. Hannan, M.A.; Akhtar, M.; Begum, R.A.; Basri, H.; Hussain, A.; Scavino, E. Capacitated vehicle-routing problem model for scheduled solid waste collection and route optimization using PSO algorithm. Waste Manag. 2018, 71, 31-41. [CrossRef] [PubMed]

28. Vasagade, T.S.; Tamboli, S.S.; Shinde, A.D. Dynamic solid waste collection and management system based on sensors, elevator and GSM. In Proceedings of the 2017 International Conference on Inventive Communication and Computational Technologies (ICICCT), Coimbatore, India, 10-11 March 2017.

29. Bueno-Delgado, M.V.; Romero-Gázquez, J.L.; Jiménez, P.; Pavón-Mariño, P. Optimal path planning for selective waste collection in smart cities. Sensors 2019, 19, 1973. [CrossRef] [PubMed]

30. Bharadwaj, A.S.; Rego, R.; Chowdhury, A. IoT based solid waste management system: A conceptual approach with an architectural solution as a smart city application. In Proceedings of the 2016 IEEE Annual India Conference (INDICON), Bangalore, India, 16-18 December 2016.

31. Misra, D.; Das, G.; Chakrabortty, T.; Das, D. An IoT-based waste management system monitored by cloud. J. Mater. Cycles Waste Manag. 2018, 20, 1574-1582. [CrossRef]

32. Shyam, G.K.; Manvi, S.S.; Bharti, P. Smart waste management using Internet-of-Things (IoT). In Proceedings of the 2017 2nd International Conference on Computing and Communications Technologies (ICCCT), Chennai, India, 23-24 February 2017. 